\title{
Working memory task performance and chunking in early Alzheimer's disease
}

\author{
Jonathan Huntley, Daniel Bor, Adam Hampshire, Adrian Owen and Robert Howard
}

\section{Background}

Chunking is a powerful encoding strategy that significantly improves working memory performance in normal young people.

\section{Aims}

To investigate chunking in patients with mild Alzheimer's disease and in a control group of elderly people without cognitive impairment.

\section{Method}

People with mild Alzheimer's disease $(n=28)$ were recruited and divided according to Mini-Mental State Examination score into mild and very mild disease groups. A control group of 15 elderly individuals was also recruited. All participants performed digit and spatial working memory tasks requiring either unstructured sequences or structured sequences (which encourage chunking of information) to be recalled.

\section{Results}

The control group and both disease groups performed significantly better on structured trials of the digit working memory tasks, indicating successful use of chunking strategies to improve verbal working memory performance. The control and very mild disease groups also performed significantly better on structured trials of the spatial task, whereas those with mild disease demonstrated no significant difference between the structured and unstructured spatial conditions.

\section{Conclusions}

The ability to use chunking as an encoding strategy to improve verbal working memory performance is preserved at the mild stage of Alzheimer's disease, whereas use of chunking to improve spatial working memory is impaired by this stage. Simple training in the use of chunking might be a beneficial therapeutic strategy to prolong working memory functioning in patients at the earliest stage of Alzheimer's disease.

\section{Declaration of interest}

None.
Although deficits in episodic memory characterise Alzheimer's disease, there is increasing evidence that working memory is also impaired at the earliest stages of the disease. ${ }^{1}$ Working memory refers to the ability to hold and manipulate information over short periods and is essential for the successful performance of many cognitive processes. Working memory deficits in Alzheimer's disease have been associated with difficulties in everyday tasks, and executive control of working memory is particularly sensitive to the effects of the disease. ${ }^{2}$ Strategic encoding of information is critical to working memory performance and learning. There is some evidence that strategic encoding is impaired at the mild stage of Alzheimer's disease but may be preserved at an earlier 'minimal' stage of the disease. ${ }^{3}$

Chunking is an effective form of strategic encoding that involves the recoding of a set of data into a compressed, efficient form and can extend working memory capacity in healthy individuals. ${ }^{4}$ A series of verbal and spatial working memory tasks have been developed that present structured sequences to encourage the reorganisation of information into higher-level chunks. Studies have demonstrated that structured stimuli significantly encourage chunking, lessening working memory demand and significantly improving working memory performance. $^{4,5}$ Training in the use of chunking strategies can lead to significant increases in working memory capacity. ${ }^{6}$ Studies of cognitive training using working memory tasks in healthy young people have demonstrated significant improvements on measures of working memory and general cognitive function, and chunking has been postulated to be a major strategy underlying these successful cognitive training regimens. ${ }^{7,8}$ It is not known, however, whether the ability to use chunking within working memory is preserved at the early stages of Alzheimer's disease. This is important, as impairment in the use of chunking may contribute to the reduction of working memory capacity noted as the disease progresses to the mild to moderate stage. Further, preserved chunking ability would provide a potential therapeutic target for cognitive training among people with Alzheimer's disease.

In this study we investigated the use of chunking strategies to improve working memory performance in people with early Alzheimer's disease. Given the evidence for preserved strategic encoding at the very mild (minimal) stage of the disease but not at the mild stage, we hypothesised that both healthy elderly participants and patients at the very mild stage of Alzheimer's disease, defined as a score above 24 on the Mini-Mental State Examination (MMSE), ${ }^{9}$ would be able to use chunking strategies to improve working memory capacity, but patients with mild Alzheimer's disease (MMSE score 18-24) would be impaired in the use of chunking strategies and therefore show no improvement in working memory capacity when given opportunities to use this strategy.

\section{Method}

Twenty-eight patients with Alzheimer's disease were recruited from the dementia case register and community mental health team (CMHT) caseloads of the South London and Maudsley National Health Service Foundation Trust. Prior to recruitment, a clinical diagnosis of Alzheimer's disease was made according to ICD-10 criteria $^{10}$ by a senior consultant psychiatrist in conjunction with a multidisciplinary team assessment. Patients also fulfilled the criteria of the National Institute of Neurological and Communicative Disorders and Stroke and the Alzheimer's Disease and Related Disorders Association (NINCDS-ADRDA Alzheimer's Criteria) for probable or possible Alzheimer's 
disease. ${ }^{11}$ All patients had demonstrated at least 2 years of deterioration in memory and some impairment of activities of daily living. Ten of 13 of the 'very mild' group and 6 of 15 of the 'mild' group were recruited from the dementia case register or had been assessed previously by researchers in the old age psychiatry department at the Institute of Psychiatry, London, and a diagnosis of Alzheimer's disease had been made prior to the study using a range of cognitive assessments, including the cognitive subscale of the Alzheimer's Disease Assessment Scale and the Logical Memory II subtest of the Wechsler Memory Scale. ${ }^{12,13}$ The remaining 3 participants in the 'very mild' group and 9 participants in the 'mild' group had all been under the care of the CMHT for at least 2 years and demonstrated progressive impairment in memory, word-finding difficulties or executive deficits and impaired activities of daily living. All participants in the two disease groups had neuroimaging (computed tomography or magnetic resonance imaging) consistent with a diagnosis of Alzheimer's disease. Patients were excluded if there was a history of head injury, cerebrovascular event, epilepsy, other major medical illness, depression or psychosis. A control group of 15 healthy individuals matched for age, gender and years of education was also recruited. Data recorded for the sample included scores on the Geriatric Depression Scale, ${ }^{14}$ years of education, IQ score derived from the National Adult Reading Test, ${ }^{15}$ and MMSE score. All participants provided written informed consent before taking part in the study, which had been approved by the Bexley and Greenwich research ethics committee.

\section{Tasks}

Two working memory span tasks were used. These were based on tasks used in previous studies investigating chunking in normal participants, ${ }^{4,5}$ and adapted for use in people with Alzheimer's disease.

\section{Digit span task}

In the digit span task a sequence of digits to be memorised appeared on a computer screen. On each trial each digit was presented for $1000 \mathrm{~ms}$ with a $500 \mathrm{~ms}$ interval between digits. At the end of the sequence a command to recall prompted the participant to recall the sequence verbally in the correct order. The recalled sequence was then typed into the computer by the experimenter, and accuracy data were collected. Trials began with a three-digit sequence and either increased by one digit if no error was made or decreased by one digit following an unsuccessful trial. In this way, sequence length tended to oscillate around the participant's maximum span capacity. In each case, average span capacity was calculated as the mean length of sequences presented in all trials. Five practice trials were given in all cases, and a total of 20 structured and 20 unstructured trials were presented in counterbalanced blocks. In the structured trials sequences to be learned were presented as runs of ascending or descending adjacent even or odd numbers, thus encouraging the recoding of digit sequences into higher-order chunks. Unstructured sequences followed no such pattern and were designed to be as random as possible (see Appendix). Previous studies have demonstrated that such structured sequences significantly encourage chunking owing to the mathematical associations inherent in structured stimuli. ${ }^{16}$

\section{Spatial span task}

Participants performed a variation of Corsi's spatial span task, ${ }^{17}$ in which they were required to memorise sequences of locations illuminated on a $4 \times 4$ grid, presented on a touch-sensitive screen.
Specifically, on each trial a sequence of red squares flashed blue, each square changing colour for $1000 \mathrm{~ms}$ with a $500 \mathrm{~ms}$ interval between squares. At the end of the sequence a short tone prompted participants to respond by touching the same series of locations with the index finger of their dominant hand. Accuracy and reaction time data were collected, although the participants were only instructed to reproduce the sequences as accurately as possible. At least ten unstructured practice trials were performed to ensure participants were able to perform the task. Twenty structured and 20 unstructured trials were then performed in counterbalanced blocks. Trials began with a three-location sequence and then either increased by one square following a successful trial or decreased by one square following an unsuccessful trial. In this way, sequence length tended to oscillate around the participant's maximum span capacity. In each case, average span capacity was calculated as the mean length of sequences presented in all 20 trials.

In the structured condition, all of the sequences followed a structured rule such that every location was in either the same column, row or diagonal as the location preceding it. In the unstructured condition two successive locations were never in the same column, row or diagonal. The result of this manipulation was subtle, in that the structured sequences tended to contain more familiar shape components, involving symmetry and parallel sides, and were thus more easily organised into higher-level patterns (see Appendix, Fig. A1). Previous studies of normal individuals have demonstrated that spatially structured trials encourage chunking into higher-level patterns. ${ }^{4}$

\section{Statistical analysis}

General linear model repeated-measures analysis was used, with structured and unstructured scores as within-participant variables and group as a between-participant variable.

\section{Results}

Demographic information on all participants is shown in Table 1. One-way analyses of variance (ANOVAs) demonstrated no significant difference in age, Geriatric Depression Scale score or years of education between the groups. There was a significant difference in IQ scores between the mild disease group and the control and very mild disease groups. The potential significance of this difference between groups is discussed below.

\section{Task performance between groups}

The participants' performance on the two tasks is summarised in Table 2.

\section{Digit span task}

General linear model repeated-measures analysis revealed a main effect of group $(F=24.8, P<0.001)$. Post hoc Bonferroni analysis demonstrated a significant difference between all groups (control $v$. very mild, $P=0.033$; control $v$. mild, $P<0.001$; very mild $v$. mild, $P=0.001$ ).

\section{Spatial span task}

Repeated-measures analysis revealed a main effect of group $(F=24.9, P<0.001)$. Post hoc Bonferroni analysis demonstrated no significant difference between the control and very mild disease groups, although this approached significance $(P=0.064)$. There was a significant difference between the mild disease group and the control $(P<0.001)$ and very mild $(P<0.001)$ disease groups. 


\begin{tabular}{|c|c|c|c|c|}
\hline & \multirow[b]{2}{*}{$\begin{array}{l}\text { Control group }(n=15) \\
\text { Mean (s.d.) }\end{array}$} & \multicolumn{2}{|c|}{ Alzheimer's disease groups } & \multirow[b]{2}{*}{$P$} \\
\hline & & $\begin{array}{l}\text { Very mild disease }(n=13) \\
\text { Mean (s.d.) }\end{array}$ & $\begin{array}{l}\text { Mild disease }(n=15) \\
\text { Mean (s.d.) }\end{array}$ & \\
\hline Age, years & $76(6.4)$ & $80(8.0)$ & 79 (7.3) & 0.297 \\
\hline MMSE score & $29.5(0.9)$ & $26.8(1.5)$ & $20.5(1.3)$ & $<0.001$ \\
\hline GDS score & $4.1(2.8)$ & $4.4(2.5)$ & $5.0(2.4)$ & 0.644 \\
\hline NART IQ score & $118.8(7.2)$ & $119.4(6.8)$ & $110.1(9.2)$ & 0.004 \\
\hline Education, years & 13.9 (3.7) & $13.3(2.5)$ & $12.2(2.6)$ & 0.316 \\
\hline
\end{tabular}

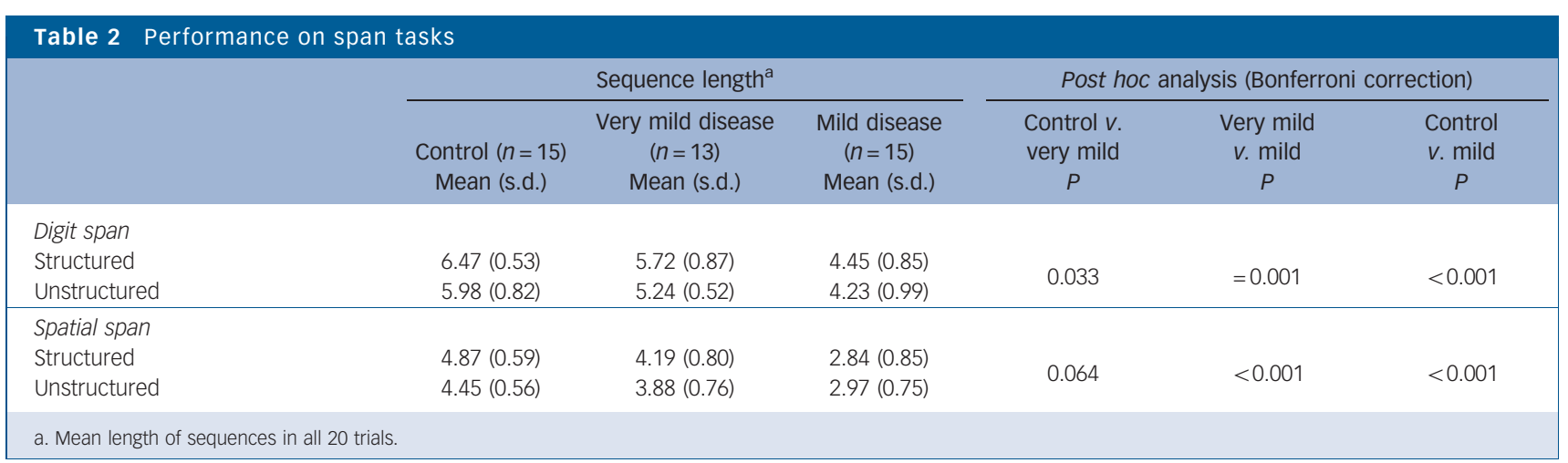

\section{Use of chunking}

Digit span task

Mean span scores for digit span tasks are presented in Fig. 1. The repeated-measures analysis revealed a main effect for structured $v$. unstructured trial type $(F=24.0, P<0.001)$ and no interaction between group and trial type $(F=1.2, P=0.309)$.

\section{Spatial span task}

Mean span scores for spatial span tasks are presented in Fig. 2. Repeated-measures analysis revealed a main effect for structured $v$. unstructured trial type $(F=8.3, P=0.006)$ and a significant interaction between group and trial type $(F=6.5, P=0.004)$. Post hoc analysis was conducted by calculating the differences between structured and unstructured trial performance for each group. Independent $t$-tests were then conducted which revealed a significant difference between the mild disease group and both the control group $(P=0.003)$ and the very mild disease group

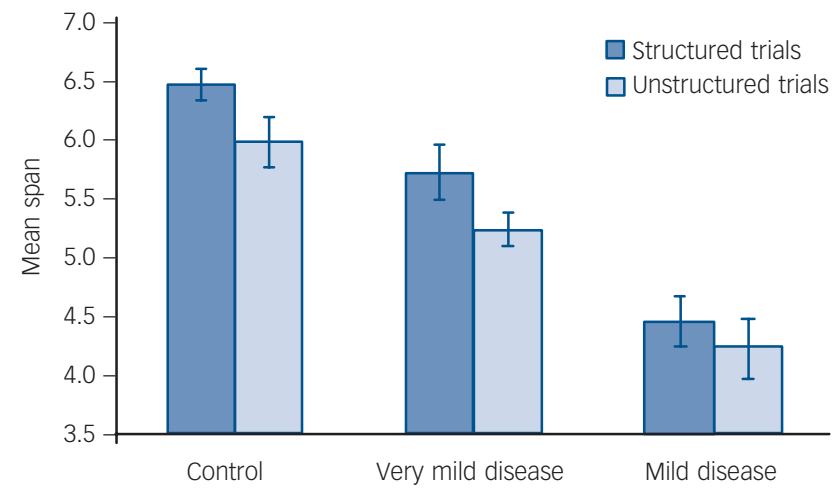

Fig. 1 Results of chunking strategy on performance of the digit span task. Error bars indicate standard errors of the means
( $P=0.012)$, with no difference between the control and very mild disease groups $(P=0.498)$.

\section{Discussion}

The main aim of this study was to identify whether patients with early Alzheimer's disease are able to use chunking to improve their working memory. The control and very mild disease groups showed improved span performance with structured sequences compared with unstructured sequences in both tasks. They were therefore able to use chunking strategies to improve verbal and spatial working memory performance. The mild disease group also demonstrated an improvement in digit span with structured compared with unstructured trials, but no advantage with structured trials in the spatial span task. This suggests that participants in the mild disease group were able to use chunking to improve digit span but not spatial span performance.

The ability to use strategic encoding strategies such as chunking in early Alzheimer's disease has been attributed to the

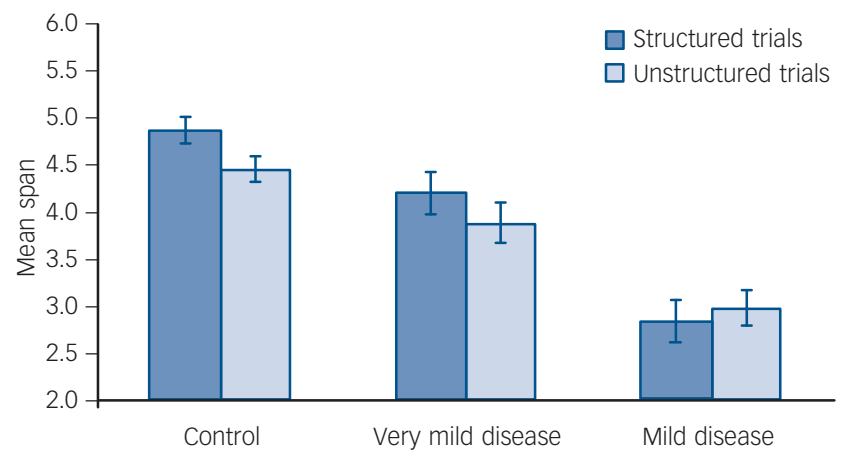

Fig. 2 Results of chunking strategy on performance of the spatial span task. Error bars indicate standard errors of the means. 
'episodic buffer' within Baddeley's working memory model. ${ }^{18}$ A previous study examining the differential performance on recall of clustered $v$. unclustered word lists, whereby associations between words in clustered lists allowed chunking, attributed the differential performance to episodic buffer functioning. ${ }^{3}$ The modulation of working memory by recoding data into higher-level chunks in our study requires the identification of mathematical rules or geometrical patterns to recode data. This in itself involves a number of higher cognitive functions including holding data in working memory, identifying mathematical associations between numbers, accessing knowledge from longterm memory stores of known shapes or mathematical rules, strategically using these associations to recode the data into a new representation in working memory and inhibiting interference from data that do not share associations with already chunked information. The finding that this ability to chunk information is impaired at the mild stage of Alzheimer's disease in spatial working memory is unsurprising given the relatively high executive demands involved. There is evidence that performance on executively demanding tasks is impaired at an early stage of Alzheimer's disease, ${ }^{19}$ with spatial span considered a more executively demanding task than digit span. ${ }^{20}$ However, contrary to our hypothesis, participants with mild Alzheimer's disease also benefited from the use of chunking in the digit span task. This result is encouraging, as it suggests that the use of mnemonic strategies in the verbal domain may remain preserved at the early stage of the disease, and may therefore be a potential target for cognitive training.

Animal and human studies have demonstrated that encoding, storage and retrieval of information in working memory are associated with activity in the prefrontal cortex and posterior parietal cortex. ${ }^{21-24}$ Functional magnetic resonance imaging (fMRI) studies have demonstrated the importance of the prefrontal cortex in working memory tasks requiring executive control. ${ }^{25} \mathrm{~A}$ confounding factor in such studies has been the observed increase in prefrontal cortex activity with increasing task difficulty. ${ }^{26}$ A series of fMRI studies using verbal and spatial chunking tasks similar to those in our study have overcome this effect of task difficulty, demonstrating activation of prefrontal cortex and posterior parietal cortex during the use of chunking strategies, despite reduced working memory demand in normal young individuals. ${ }^{4,5}$ There is evidence that neuropathological changes in Alzheimer's disease result in reduced effective connectivity between brain regions, including prefrontal and parietal regions ${ }^{27}$ identified as being involved in chunking. Functional imaging studies have implicated a range of areas in spatial working memory, including frontal, posterior parietal and occipital cortex. ${ }^{28}$ Such disconnectivity between these areas may therefore underlie the impairment observed in the use of spatial chunking strategies at an early stage of Alzheimer's disease.

\section{Group performance comparison}

In the mild Alzheimer's disease group, performance on verbal and spatial working memory span tasks was impaired compared with the control and very mild disease groups. Similar results have been reported from previous studies of verbal and spatial working memory in mild Alzheimer's disease, ${ }^{29,30}$ although some studies have found no impairment in mild disease. ${ }^{31,32}$ At the very mild stage of Alzheimer's disease performance on spatial span tasks was not significantly impaired, although this approached significance $(P=0.064)$, whereas digit span performance was impaired in these participants $(P=0.033)$. Previous studies of people with 'minimal' Alzheimer's disease and minor cognitive impairment have suggested that performance on span tasks remains intact at the preclinical stage. ${ }^{32,33}$ Our findings that our very mild disease group were impaired on digit span performance might be due to their greater cognitive impairment compared with preclinical samples in other studies.

According to the model of working memory proposed by Baddeley \& Hitch, successful performance on working memory span tasks relies on the functioning of subsidiary 'slave' systems (phonological loop for verbal information, and visuospatial sketchpad for visuospatial information) and central executive functioning. ${ }^{34}$ Central executive functioning is impaired earlier in Alzheimer's disease than the subsidiary systems, which may remain relatively intact until the disease is at the mild to moderate stage. ${ }^{35}$ Our finding that performance on verbal and spatial working memory tasks is significantly impaired by the mild stage of Alzheimer's disease may therefore reflect impairments in central executive functioning rather than in the subsidiary systems.

\section{Chunking in cognitive training}

Training in the use of chunking strategies can significantly increase working memory capacity. ${ }^{6}$ We observed that some participants in the control and very mild disease groups were not explicitly aware of using chunking strategies, despite successfully doing so. Following initial testing, a small subset of four patients with very mild Alzheimer's disease and one with mild Alzheimer's disease were explicitly informed about chunking techniques and retested on the structured digit span task, to examine whether working memory performance improved in a single session. This was the case for those with very mild disease - the mean digit span improved from 5.4 (s.d. $=1.27$ ) to 6.1 (s.d. $=0.8 ; P=0.08$ ) - but not for the patient with mild disease. This finding, together with evidence of the efficacy of chunking in cognitive training in normal young individuals, ${ }^{7,8}$ suggests that simple training in chunking techniques may be a useful cognitive training strategy for improving working memory performance in people with very mild Alzheimer's disease.

\section{Benefits of cognitive training:}

A recent large study of brain training in healthy adults (aged 1860 years) demonstrated no evidence that such training led to any generalised improvement in cognitive functioning; significant improvements were seen on the trained tasks but generalisation did not occur even to untrained tasks that required similar cognitive functions. ${ }^{36}$ However, there is some evidence that cognitive training is effective in older people. ${ }^{37}$ There is also some evidence of benefit from cognitive training in people with Alzheimer's disease or mild cognitive impairment. ${ }^{38,39}$ It may be the case that, whereas healthy young people already perform at their full capacity, elderly individuals or those with cognitive impairment would benefit from learning or relearning specific strategies to help compensate for their underlying cognitive deterioration and loss of inherent strategic abilities. There is evidence that cognitive training using restorative strategies may be more efficacious than compensatory strategies in both cognitive domains and everyday functioning. ${ }^{40}$ Many uncertainties remain as to the benefits of cognitive training in Alzheimer's disease. It is unclear how sustained any benefits of cognitive training would be in the face of continued neurodegeneration; however, our results provide a case for studies that investigate the use of and training in well-defined mnemonic strategies such as chunking in the growing population of individuals with early Alzheimer's disease. 


\section{Methodological issues}

We divided our Alzheimer's disease patients into two groups according to their MMSE score. The MMSE is commonly used as a screening tool for dementia, and has been used in several other studies as a marker for severity of Alzheimer's disease.,32 However, objections have been raised as to whether it is suitable for this purpose. ${ }^{41}$ Individuals with a high premorbid level of functioning and educational achievement can score relatively well on the MMSE despite having significant dementia. More detailed neuropsychological assessment would undoubtedly have been useful in classifying the severity of dementia in our participants. Although we acknowledge this, the MMSE served as a useful clinical marker of Alzheimer's disease severity to test our hypothesis. We are not suggesting that our very mild disease group represents a homogeneous clinical entity, but rather that in this study it represented a group of patients who were at an earlier stage of disease progression than those in our mild disease group. We were interested in examining whether patients with mild Alzheimer's disease were able to use chunking to improve working memory performance and whether this ability was lost during the early stages of the disease. We therefore used MMSE scores as a pragmatic cut-off to test this hypothesis, not as a diagnostic marker or to suggest a homogeneous clinical population.

Our finding that chunking ability appears preserved in patients who score above 24 on the MMSE is in keeping with other studies that examined executive function at such an early stage in the disease; ${ }^{3}$ however, further larger studies incorporating more detailed information about specific attentional and executive deficits would be helpful.

IQ scores

Although all participants were recruited from the same geographical area and matched for age, gender and years of education, analysis of NART scores revealed a significant difference between the mild disease group and the very mild disease and control groups. Subsequent repeated-measures analysis using IQ as a covariable revealed no significant interaction between structured $v$. unstructured trial type and IQ $(F=0.6$, $P=0.438)$ for either the digit or the spatial span task. Bivariate correlation analysis revealed a highly significant correlation between IQ and MMSE scores (Pearson correlate 0.528, $P<0.01)$. Although the NART is often used to assess premorbid IQ, its validity in dementia has been questioned, as the cognitive deficits may lead to poor performance on this measure. It has been demonstrated that NART score is impaired even at mild stages of the disease, ${ }^{42}$ and this is reflected in the high correlation in our study between IQ and MMSE scores. The difference in IQ scores seen in our study between the mild disease group and the other two groups is therefore likely to reflect the cognitive impairment caused by Alzheimer's disease, rather than representing a true difference in premorbid IQ between the groups.

\section{Study implications}

We conclude that healthy elderly people and people with very mild Alzheimer's disease are able to use chunking strategies to improve working memory in both verbal and spatial domains, whereas those with mild Alzheimer's disease remain able to benefit from chunking in verbal but not spatial working memory. Chunking is a well-established form of strategic encoding that has been shown to improve working memory capacity and underlie improvements seen in cognitive training programmes. Our study opens up the possibility that training in the use of chunking strategies might be a simple but effective tool to help maintain working memory at the early stages of Alzheimer's disease.
Jonathan Huntley, MRCPsych, Department of Old Age Psychiatry, Institute of Psychiatry, King's College London; Daniel Bor, PhD, Sackler Centre for Consciousness Science, University of Sussex; Adam Hampshire, PhD, Adrian Owen, PhD, Medical Research Council Cognition and Brain Sciences Unit, Cambridge; Robert Howard, MD, MRCPsych, Department of Old Age Psychiatry, Institute of Psychiatry, King's College London, London, UK

Correspondence: Jonathan D. Huntley, Department of Old Age Psychiatry, Institute of Psychiatry, King's College London, PO Box 70, London SE5 8AF, UK. Email: jonathan.huntley@kcl.ac.uk

First received 17 Jun 2010, final revision 20 Oct 2010, accepted 20 Dec 2010

\section{Appendix}

\section{Examples of verbal and spatial span tasks}

The following examples of verbal and spatial span tasks illustrate the differences between structured trials, which encourage chunking, and unstructured trials.

verbal span task

Structured sequence:

\section{5}

Unstructured sequence:

816294

\section{Spatial span task}

An example of a structured sequence is shown in Fig. A1(a) and an unstructured form in Fig. A1(b). (a)

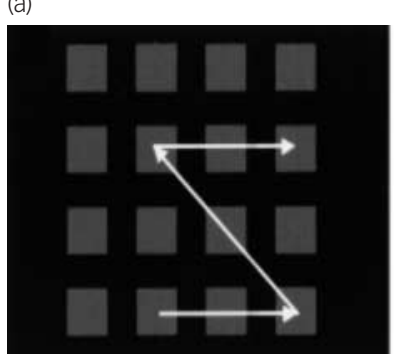

(b)

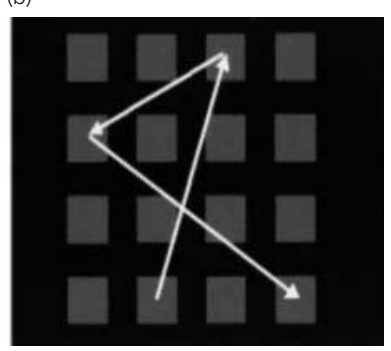

Fig. A1 Spatial span task: (a) structured; (b) unstructured. Reprinted from Bor et $a l^{4}$ with permission from Elsevier.

\section{References}

1 Huntley JD, Howard RJ. Working memory in early Alzheimer's disease: a neuropsychological review. Int J Geriatr Psychiatry 2010; 25: 121-32.

2 Belleville S, Chertkow H, Gauthier S. Working memory and control of attention in persons with Alzheimer's disease and mild cognitive impairment. Neuropsychology 2007; 21: 458-69.

3 Germano C, Kinsella GJ, Storey E, Ong B, Ames D. The episodic buffer and learning in early Alzheimer's disease. J Clin Exp Neuropsychol 2008; 30: 62738

4 Bor D, Duncan J, Wiseman RJ, Owen AM. Encoding strategies dissociate prefrontal activity from working memory demand. Neuron 2003; 37: 361-7.

5 Bor D, Cumming N, Scott CE, Owen AM. Prefrontal cortical involvement in verbal encoding strategies. Eur J Neurosci 2004; 19: 3365-70.

6 Ericcson KA, Chase WG, Faloon S. Acquisition of a memory skill. Science 1980; 208: 1181-2.

7 Olesen PJ, Westerberg $\mathrm{H}$, Klingberg $\mathrm{T}$. Increased prefrontal and parietal activity after training of working memory. Nat Neurosci 2004; 7: 75-9.

8 McNab F, Varrone A, Farde L, Jucaite A, Bystritsky P, Forssberg $H$, et al. Changes in cortical dopamine D1 receptor binding associated with cognitive training. Science 2009; 323: 800-2.

9 Folstein MF, Folstein SE, McHugh PR. 'Mini-mental state'. A practical method for grading the cognitive state of patients for the clinician. J Psychiatr Res 1975; 12: 189-98. 
10 World Health Organization. International Statistical Classification of Diseases and Related Health Problems (ICD-10). WHO, 1992.

11 McKhann G, Drachman D, Folstein M, Katzman R, Price D, Stadlan EM. Clinical diagnosis of Alzheimer's disease: report of the NINCDS-ADRDA Work Group under the auspices of Department of Health and Human Services Task Force on Alzheimer's Disease. Neurology 1984; 34: 939-44.

12 Rosen WG, Mohs RC, Davis KL. A new rating scale for Alzheimer's disease. Am J Psychiatry 1984; 141: 1356-64.

13 Wechsler D. Wechsler Memory Scale (3rd edn). Psychological Corporation, 1998.

14. Yesavage JA, Brink TL, Rose TL, Lum O, Huang V, Adey M, et al. Development and validation of a geriatric depression screening scale: a preliminary report. J Psychiatr Res 1983; 17: 37-49.

15 Nelson H, Willison J. National Adult Reading Test. nferNelson, 1991.

16 Bor D, Owen AM. A common prefrontal-parietal network for mnemonic and mathematical recoding strategies within working memory. Cereb Cortex 2007; 17: 778-86.

17 Milner B. Interhemispheric differences in the localization of psychological processes in man. Br Med Bull 1971; 27: 272-7.

18 Baddeley A. The episodic buffer: a new component of working memory? Trends Cogn Sci 2000; 4: 417-23.

19 Perry RJ, Hodges JR. Attention and executive deficits in Alzheimer's disease. A critical review. Brain 1999; 122: 383-404.

20 Carlesimo GA, Fadda L, Lorusso S, Caltagirone C. Verbal and spatial memory spans in Alzheimer's and multi-infarct dementia. Acta Neurol Scand 1994; 89: $132-8$.

21 Goldman-Rakic PS. The prefrontal landscape: implications of functional architecture for understanding human mentation and the central executive. In The Prefrontal Cortex: Executive and Cognitive Functions (eds AC Roberts, TW Robbins, L Weiskrantz): 87-102. Oxford University Press, 1998.

22 Miller EK, Cohen JD. An integrative theory of prefrontal cortex function. Annu Rev Neurosci 2001; 24: 167-202.

23 Owen AM. The functional organization of working memory processes within human lateral frontal cortex: the contribution of functional neuroimaging. Eur J Neurosci 1997; 9: 1329-39.

24 Cabeza R, Nyberg L. Imaging cognition II: an empirical review of 275 PET and fMRI studies. J Cogn Neurosci 2000; 12: 1-47.

25 Owen AM, McMillan KM, Laird AR, Bullmore E. N-back working memory paradigm: a meta-analysis of normative functional neuroimaging studies. Hum Brain Mapp 2005; 25: 46-59.

26 Duncan J, Owen AM. Common regions of the human frontal lobe recruited by diverse cognitive demands. Trends Neurosci 2000; 23: 475-83.
27 Delbeuck $X$, Van der Linden $M$, Collette F. Alzheimer's disease as a disconnection syndrome? Neuropsychol Rev 2003; 13: 79-92.

28 Smith EE, Jonides J. Neuroimaging analyses of human working memory. Proc Natl Acad Sci U S A 1998; 95: 12061-8.

29 Becker JT. Working memory and secondary memory deficits in Alzheimer's disease. J Clin Exp Neuropsychol 1988; 10: 739-53.

30 Hodges JR, Patterson K. Is semantic memory consistently impaired early in the course of Alzheimer's disease? Neuroanatomical and diagnostic implications. Neuropsychologia 1995; 33: 441-59.

31 Lines CR, Dawson C, Preston GC, Reich S, Foster C, Traub M. Memory and attention in patients with senile dementia of the Alzheimer type and in normal elderly subjects. J Clin Exp Neuropsychol 1991; 13: 691-702.

32 Perry RJ, Watson P, Hodges JR. The nature and staging of attention dysfunction in early (minimal and mild) Alzheimer's disease: relationship to episodic and semantic memory impairment. Neuropsychologia 2000; 38: 252-71.

33 Traykov L, Raoux N, Latour F, Gallo L, Hanon O, Baudic S, et al. Executive functions deficit in mild cognitive impairment. Cogn Behav Neurol 2007; 20 219-24.

34 Baddeley AD, Hitch GJ. Working memory. In The Psychology of Learning and Motivation: Advances in Research and Theory (ed GH Bower): 48-89. Academic Press, 1974.

35 Collette F, Van der Linden M, Bechet S, Salmon E. Phonological loop and central executive functioning in Alzheimer's disease. Neuropsychologia 1999; 37: 905-18.

36 Owen AM, Hampshire A, Grahn JA, Stenton R, Dajani S, Burns AS, et al. Putting brain training to the test. Nature 2010; 465: 775-8.

37 Willis SL, Tennstedt SL, Marsiske M, Ball K, Elias J, Koepke KM, et al. Long term effects of cognitive training on everyday functional outcomes in older adults. JAMA 2006; 296: 2805-14.

38 Bier N, van der Linden M, Gagnon L, Desrosiers J, Adam S, Louveaux S, et al. Face-name association learning in early Alzheimer's disease: a comparison of learning methods and their underlying mechanisms. Neuropsychol Rehabil 2008; 18: 343-71.

39 Belleville S. Cognitive training for persons with mild cognitive impairment. Int Psychogeriatr 2008; 20: 57-66.

40 Sitzer DI, Twamley EW, Jeste DV. Cognitive training in Alzheimer's disease: a meta-analysis of the literature. Acta Psychiatr Scand 2006; 114: 75-90.

41 Greene JD, Baddeley AD, Hodges JR. Analysis of the episodic memory deficit in early Alzheimer's disease: evidence from the doors and people test. Neuropsychologia 1996; 34: 537-51.

42 McFarlane J, Welch J, Rodgers J. Severity of Alzheimer's disease and effect on premorbid measures of intelligence. Br J Clin Psychol 2006; 45: 453-63. 